\title{
PERCEPCIÓN DE LAS COMUNIDADES DEL TERRITORIO DEL CONSEJO COMUNITARIO DE LA PLATA BAHÍA MÁLAGA FRENTE A LAVULNERABILIDAD AL CAMBIO CLIMÁTICO
}

\author{
Perception of Communities the Territory Community Council \\ La Plata Bahia Malaga Front to Vulnerability to Climate Change
}

\author{
HELEM ALEXANDER RUÍZ1,2 \\ ${ }^{1}$ Funcionario; Corporación Autónoma Regional del Valle del Cauca CVC. \\ 2Maestría en Desarrollo Sostenible en Desarrollo y Medio Ambiente, Universidad de Manizales.
}

E-mail: halexruiz13@gmail.com

Recibido: 11 de Noviembre de 2014

Aceptado: 5 de Diciembre de 2014

\section{Resumen}

En los próximos años según las proyecciones mundiales sobre el cambio climático, las comunidades rurales serán las más afectadas por los efectos de este fenómeno, no solo en su composición territorial, sino en sus recursos vitales; los efectos del cambio climático ya no están lejos de nosotros, y sus efectos son cada vez más visibles. Si bien el país ha optado por el camino de la adaptación y mitigación, tener conocimiento de las vulnerabilidades en los territorios, es una ruta muy importante en el propósito de asimilar las afectaciones producidas por el cambio climático. El presente manuscrito, pretende dar a conocer el estado de la vulnerabilidad de la comunidad del territorio colectivo de la comunidad negra de La Plata Bahía Málaga, comunidad rural del Distrito de Buenaventura, desde la percepción local de sus líderes, dándole importancia a los saberes y percepciones de los habitantes, desde una visión intergeneracional, que permita comprender desde su cosmovisión la vulnerabilidad de las comunidades que conforman el consejo comunitario.

Palabras claves: Amenaza, Consejo Comunitario, Cambio climático, Territorio colectivo, Manglar, Vulnerabilidad.

\section{Abstract}

In the coming years as global projections of climate change, rural communities will be most affected by the effects of this phenomenon, not only in its territorial composition, but its vital resources; the effects of climate change are already not far from us, and its effects are increasingly visible, although the country has chosen the path of adaptation and mitigation, have a knowledge of the vulnerabilities in the territories, is a route very important in the way of assimilating the damages caused by climate change. This writing, seeks to highlight the status of the vulnerability of the community the collective territory of the black community of La Plata Bahia Malaga, from local perceptions of their leaders, giving importance to knowledge and perceptions of the people, from a intergenerational vision for understanding their worldview from the vulnerability of the communities that make up the council.

Keywords: Threats, Community Council, Climate change, Collective territory, Mangrove, Vulnerability. 


\section{INTRODUCCIÓN}

La Universidad de Manizales, a través del Centro de Investigaciones en Medio Ambiente y Desarrollo Sostenible, y la Maestría en Desarrollo Sostenible y Medio Ambiente, desarrolló la investigación "Estado y prospectiva de las posibilidades de adaptación y mitigación de impacto del cambio climático en diferentes regiones del país". Este proyecto de investigación tuvo como objetivo general: analizar e interpretar las acciones que se vienen realizando en diferentes regiones del país, para mitigar los efectos adversos del cambio climático y la capacidad de adaptación de las diversas regiones para afrontar las situaciones derivadas de dicho cambio, con el objetivo último de reducir la vulnerabilidad de la población ante este fenómeno de índole global. Esta investigación apuntó a describir tres categorías centrales de investigación: Adaptación, mitigación y vulnerabilidad, asociadas al cambio climático, con una perspectiva integral e interdisciplinar, que busca ser un aporte al entendimiento de la problemática en Colombia.

En aras de aportar a la construcción de este importante esfuerzo; como un capítulo especial a este proyecto, se incluyó un caso particular de la región del Pacífico vallecaucano, específicamente en el territorio del Consejo Comunitario de La Plata Bahía Málaga, Buenaventura, donde se analizaron las tres categorías antes mencionadas (adaptación, mitigación y vulnerabilidad) en relación al territorio. Este capítulo buscó tener una visión local del cambio climático y así dar una referencia documental de las percepciones de la problemática en estas comunidades.

Producto de la realización del proyecto marco y de este capítulo especial en el territorio del consejo comunitario de la plata Bahía Málaga se presenta aquí un manuscrito con base a los resultados alcanzados, en relación a la categoría de vulnerabilidad.

\section{Aspectos generales de la vulnerabilidad de Colombia ante el cambio climático}

La segunda comunicación nacional de Colombia ante la CMNUCC, muestra los avances del país en varios aspectos, mitigación, adaptación y vulnerabilidad, en esta última, se evidencia lo expuesto que se encuentra gran parte del territorio colombiano a los impactos del cambio climático, estas vulnerabilidades están determinadas en diferentes zonas como son: Orobioma alto andino, cuerpos de agua, áreas protegidas, impacto en sector agropecuario y áreas de minifundio campesino debido a la reducción de precipitación; bosques, ecosistemas secos, áreas con infraestructura para generación hidroeléctrica y las zonas costeras e insulares; para estas últimas se esperan las siguientes consecuencias:

Para el año 2100 un ascenso del nivel del mar en un metro, la población que se vería afectada estaría por el orden de 1,4 a 1,7 millones de habitantes, equivalentes entre dos y tres por ciento de la población nacional en ese mismo año; de estos porcentajes, $80 \%$ corresponde al Caribe y $20 \%$ al Pacífico (IDEAM 2010).

Aproximadamente $55 \%$ de la población del litoral Caribe estará expuesta a los efectos directos de la inundación marina (IDEAM 2010). De la población afectada, aproximadamente $90 \%$ se encuentra localizada en las cabeceras municipales, en tanto que la restante se distribuye en forma dispersa en las áreas rurales (IDEAM 2010).

En el litoral Pacífico, alrededor de $41 \%$ de la población sería afectada por la inundación marina debida al ascenso del nivel del mar (IDEAM 2010). De dicha población afectada, aproximadamente $36 \%$ se encuentra localizada en cabeceras municipales, en tanto que la población restante se distribuye en forma dispersa en el sector rural (IDEAM 2010). Las cabeceras municipales de Tumaco, El Charco, Nuquí, Juradó, Santa Bárbara y Olaya Herrera son las que más población urbana concentran en las zonas con alguna posibilidad de inundación (IDEAM 2010).

Con el ascenso del nivel del mar en un metro se podría causar una inundación estimada, mayor del $10 \%$ de la isla de San Andrés, representado en áreas de marismas, cordones litorales, rellenos artificiales y algunas terrazas coralinas bajas, cubiertas por mangle. En estas áreas se verían afectadas zonas urbanas de uso residencial y comercial, así como el puerto de la isla. En las islas de Providencia y Santa Catalina, el área expuesta a la inundación representa 3,8\% del área de las islas, donde se incluyen sectores actualmente ocupados por zonas residenciales, comerciales y públicas, entre las cuales se encuentra el puerto de Providencia (IDEAM 2010).

Como se ha comentado, esta información muestra condiciones generales de las regiones, con base en la evaluación de los modelos climáticos globales, tales como CCSM (CCSM Community Climate System Model) para el modelo global GSM (Global Spectral Model) de alta resolución del MRI 
Ambiente y Sostenibilidad 2014 (4): 51-64

Revista del Doctorado Interinstitucional en Ciencias Ambientales

ISSN: 2339-3122

(Meteorological Research Institute),que mejor representan el clima regional y con la ayuda de modelos climáticos regionales de alta resolución espacial, como los modelos PRECIS (ProvidingRegional Climates for impact Studies) de Reino Unido y WRF (Weather Research and Forecast) de los Estados Unidos; los cuales usan física aplicable al sistema climático, permitiendo modelar directamente la dinámica del sistema físico que caracteriza el clima de una región (IDEAM 2010).

Estos modelos permiten tomar decisiones a nivel macro, pero es muy poco lo que se puede evidenciar de las comunidades locales y de los efectos del cambio climático en sus territorios, que permitirían tomar acciones más eficientes que son de mucha importancia para la solución de las problemáticas asociadas a los efectos del cambio climático (mitigación, vulnerabilidad y adaptación).

\section{CARACTERIZACIÓN DEL CONSEJO COMUNITARIO DE LA PLATA BAHÍA MÁLAGA}

En el año de 1993 el gobierno nacional a través de la ley 70 y en cumplimiento de lo establecido en el artículo transitorio 55 de la Constitución nacional de 1993, reconoce a las comunidades negras el derecho a la propiedad colectiva de las tierras baldías que han venido ocupando en las zonas rurales ribereñas de los ríos de la Cuenca del Pacífico, y en otras zonas del país.

Basado en estas disposiciones legales gran parte de las comunidades negras del Colombia se establecieron como consejos comunitarios, que como lo define el decreto 1745 de 1995 en su artículo 3 son la máxima autoridad de administración interna dentro de las tierras de las Comunidades Negras, de acuerdo con los mandatos constitucionales y legales que los rigen y los demás que le asigne el sistema de derecho propio de cada comunidad". En los términos del numeral $5^{\circ}$, artículo $2^{\circ}$ de la Ley 70 de 1993, Comunidad Negra es el conjunto de familias de ascendencia afrocolombiana que poseen una cultura propia, comparten una historia y tienen sus propias tradiciones y costumbres dentro de la relación campo-poblado, que revelan y conservan conciencia e identidad que las distinguen de otros grupos étnicos.

Al Consejo Comunitario lo integran la Asamblea General y la Junta del Consejo Comunitario (Decreto 1745 de 1995).
Con estas herramientas nace en el año 2003 el Consejo Comunitario de la Plata Bahía Málaga, como un territorio colectivo de las comunidades negras, que cuenta con más de trescientos años de tradición y presencia en este territorio, el cual lo ha conservado con base a sus prácticas tradicionales, espiritualidad y aprovechamiento de los recursos naturales, administrándolo y protegiéndolo (Código de Régimen Interno, C.C de La Plata Bahía Málaga 2012).

El territorio titulado del consejo comunitario La Plata- Bahía Málaga se encuentra ubicado en las coordenadas descritas a continuación : Punto $1: 4^{\circ} 8.471^{\prime}$ Norte, $77^{\circ} 13.080^{\prime}$ Oeste, Punto 2: $4^{\circ} 07.609^{\prime}$ Norte, $77^{\circ} 10.161^{\prime}$; Oeste, Punto 3: $4^{\circ} 01.609^{\prime}$ Norte, $77^{\circ} 06.989^{\prime}$ Oeste.; Punto 4: $4^{\circ} 02.781^{\prime}$ Norte, $77^{\circ} 10.469^{\prime}$; Punto 5: $4^{\circ} 04.251^{\prime}$ Norte, $77^{\circ} 12.930^{\prime}$ Oeste; Punto $6: 4^{\circ} 07.203^{\prime}$ Norte, $77^{\circ} 12.930^{\prime}$. Globo de Ampliación 1: Punto 1: $4^{\circ} 08.449^{\prime}$ Norte, $77^{\circ} 15.379^{\prime}$ Oeste; Punto 2: $4^{\circ} 07.528^{\prime}$ Norte, $77^{\circ} 14.170^{\prime}$ Oeste; Punto 3: $4^{\circ} 07.226^{\prime}$ Norte, $77^{\circ} 13.172^{\prime}$ Oeste; Punto 4: $4^{\circ} 07.203^{\prime}$ Norte, $\quad 77^{\circ} 12.930^{\prime}$ Oeste; Punto 5: $4^{\circ} 04.251^{\prime}$ Norte, 77¹2.930'Oeste; Punto 6: $4^{\circ} 03.894^{\prime}$ Norte, $77^{\circ} 12.810^{\prime}$ Oeste; Punto 7: $4^{\circ} 04.988^{\prime}$ Norte, $77^{\circ} 15.040^{\prime}$ Oeste; Punto 8: $4^{\circ} 06.092^{\prime}$ Norte, $77^{\circ} 17.319^{\prime}$ Oeste; Punto 9: $4^{\circ} 06.846^{\prime}$ Norte, 77¹7.035'Oeste; Punto 10: $4^{\circ} 08.449^{\prime}$ Norte, $77^{\circ} 15.379^{\prime}$ Oeste. Globo de ampliación 2: Punto 1: $4^{\circ} 02.787^{\prime}$ Norte, 77²12.226'Oeste; Punto 2: $4^{\circ} 02.687^{\prime}$ Norte, $77^{\circ} 10.373^{\prime}$ Oeste; Punto 3: $3^{\circ} 56.777^{\prime}$ Norte, $76^{\circ} 59.328^{\prime}$ Oeste; Punto 4: $3^{\circ} 56.529^{\prime}$ Norte, $76^{\circ} 00.147^{\prime}$ Oeste; Punto 5: $3^{\circ} 57.412^{\prime}$ Norte, 7703.219'Oeste; Punto 6: $3^{\circ} 55.804^{\prime}$ Norte, $7^{\circ} 07.144^{\prime}$ Oeste; Punto 7: $3^{\circ} 53.643^{\prime}$ Norte, $77^{\circ} 14.888^{\prime}$ Oeste; Punto 8: $3^{\circ} 53.927^{\prime}$ Norte, $77^{\circ} 15.669^{\prime}$ Oeste; Punto 9: $3^{\circ} 54.853^{\prime}$ Norte, $77^{\circ} 15.447^{\prime}$ Oeste; Punto 10: $3^{\circ} 55.940^{\prime}$ Norte, $77^{\circ} 16.518^{\prime}$ Oeste; Punto 11: $3^{\circ} 57.373^{\prime}$ Norte, $77^{\circ} 16.481^{\prime}$ Oeste.

Al consejo comunitario de La Plata Bahía Málaga se puede acceder por vía terrestre o marítima, siendo la principal vía de acceso la marítima, la distancia en tiempo depende de las condiciones climáticas, tipo de motor y la clase de embarcación, pero se puede estimar en unas dos a tres horas aproximadamente desde Buenaventura (Figuras 1 y 2).

\section{CONDICIONES GEOGRÁFICAS, POLÍTICAS, ECONÓMICAS Y CULTURALES DEL CONSEJO COMUNITARIO DE LA PLATA-BAHÍA MÁLAGA}

El territorio del Consejo Comunitario de la Plata Bahía Málaga tiene una extensión de 38037 has $+1364 \mathrm{~m}^{2}$, y está conformado 
por las veredas de La Sierpe, la vereda La Plata, Mangaña y Miramar; presenta una temperatura promedio anual de 25.7 ${ }^{\circ} \mathrm{C}$, la humedad relativa está dada en un $89.7 \%$ y su altura sobre el nivel del mar va de cero 0 a 100 m.s.n.m., se calcula que las precipitaciones promedio anuales son generalmente superiores a $6.000 \mathrm{~mm}$, y en muchos casos alcanzan los $7.000 \mathrm{e}$ incluso los $8.000 \mathrm{~mm}$ (CENIPACÍFICO 1986). La topografía del área está conformada por colinas denudativas; altas y bajas, algunas de ellas muy disectadas y alargadas, otras con crestas paralelas; las pendientes varían de suaves a escarpadas (3-7 \% y $25-50 \%)$, las geoformas asociadas a este territorio son; las colinas y montañas, terrazas y depósitos aluviales antiguos levantados, barras y bajos arenosos, algunas playas y depósitos de conos aluviales (INVEMAR et al. 2006).

La Biodiversidad presente en el territorio del Consejo Comunitario de la Plata Bahía Málaga es alta, existen entre 8000 y 9000 especies de plantas de las 45000 que existen en Colombia, registrando uno de los mayores índices de endemismos continental de plantas y aves (CVC \& C.C La Plata Bahía Málaga 2009). En cuanto a fauna, se presenta la región como un centro de diversidad y endemismo de mariposas, la zona de mayor diversidad de anfibios; se estima que en la región está representado el $4.5 \%$ de especies de herpetofauna ( $45 \%$ para el país) y $11 \%$ de todas las especies conocidas en el mundo de aves albergando especies tanto de agua dulce como marinas (CVC \& C.C La Plata Bahía Málaga 2009).

Los ecosistemas presentes en el territorio del Consejo Comunitario se encuentran clasificados en el Pedobioma llamado Halobioma del Pacífico, en este sitio se presentan dos grandes bloques: Los ecosistemas continentales y los ecosistemas marinos costeros, los primeros corresponden al Bosque Húmedo Tropical, las quebradas y riachuelos y los segundos incluyen los manglares, estuarios, islas e islotes, las playas arenosas, los litorales rocosos-acantilados, los Bentos, Pelágicos Nerítico y Bentónico Nerítico. (Galindo et al. 2009).
El consejo comunitario de la Plata - Bahía Málaga tiene una población de 573 habitantes, compuesta por 111 familias. Este Consejo Comunitario por su condición de territorio colectivo, se encuentra conformado por comunidades campesinas que pertenecen al estrato social de nivel uno, con un nivel de escolaridad en promedio de cuarto de primaria (Código de Régimen interno C. C de la Plata -Bahía Málaga 2012), en su mayoría tienen la propiedad sobre sus viviendas.

De acuerdo con el censo general 2005, actualizado en noviembre 2010, el municipio de Buenaventura en su zona rural, presenta un nivel de hacinamiento del $24 \%$. La densidad poblacional promedio del municipio de Buenaventura es de 45.2 habitante $/ \mathrm{km}^{2}$, de acuerdo a los datos establecidos por el DANE (2005) y Planeación departamental (2010); para el caso del territorio del Consejo Comunitario la densidad poblacional es de 1.51 habitantes/ $\mathrm{km}^{2}$, lo cual muestra lo poco poblado del territorio y el gran área natural que existe. La cobertura en agua potable y alcantarillado es muy baja, casi nula, esto debido a que tanto el gobierno nacional como el municipal, realizan muy pocas inversiones en las comunidades rurales, los pocos aportes son dados por entidades extranjeras y en alguna medida por la Corporación Autónoma Regional del Valle del Cauca CVC.

El Índice de Desarrollo Humano, no ha sido calculado para esta comunidad en particular, sin embargo, para la zona rural del municipio de Buenaventura el promedio de los últimos años es de 0,58 (DANE et al. 2005), en materia de pobreza los valores no son alentadores y el índice de pobreza para el municipio de Buenaventura de acuerdo a la encuesta de hogares realizada en el 2003 por el municipio de manera conjunta con el DANE, demuestran que la incidencia de la pobreza alcanza el $80,6 \%$ por ingresos y $62.7 \%$ por necesidades básicas insatisfechas (DANE 2003). 
Ambiente y Sostenibilidad 2014 (4): 51-64

Revista del Doctorado Interinstitucional en Ciencias Ambientales

ISSN: 2339-3122

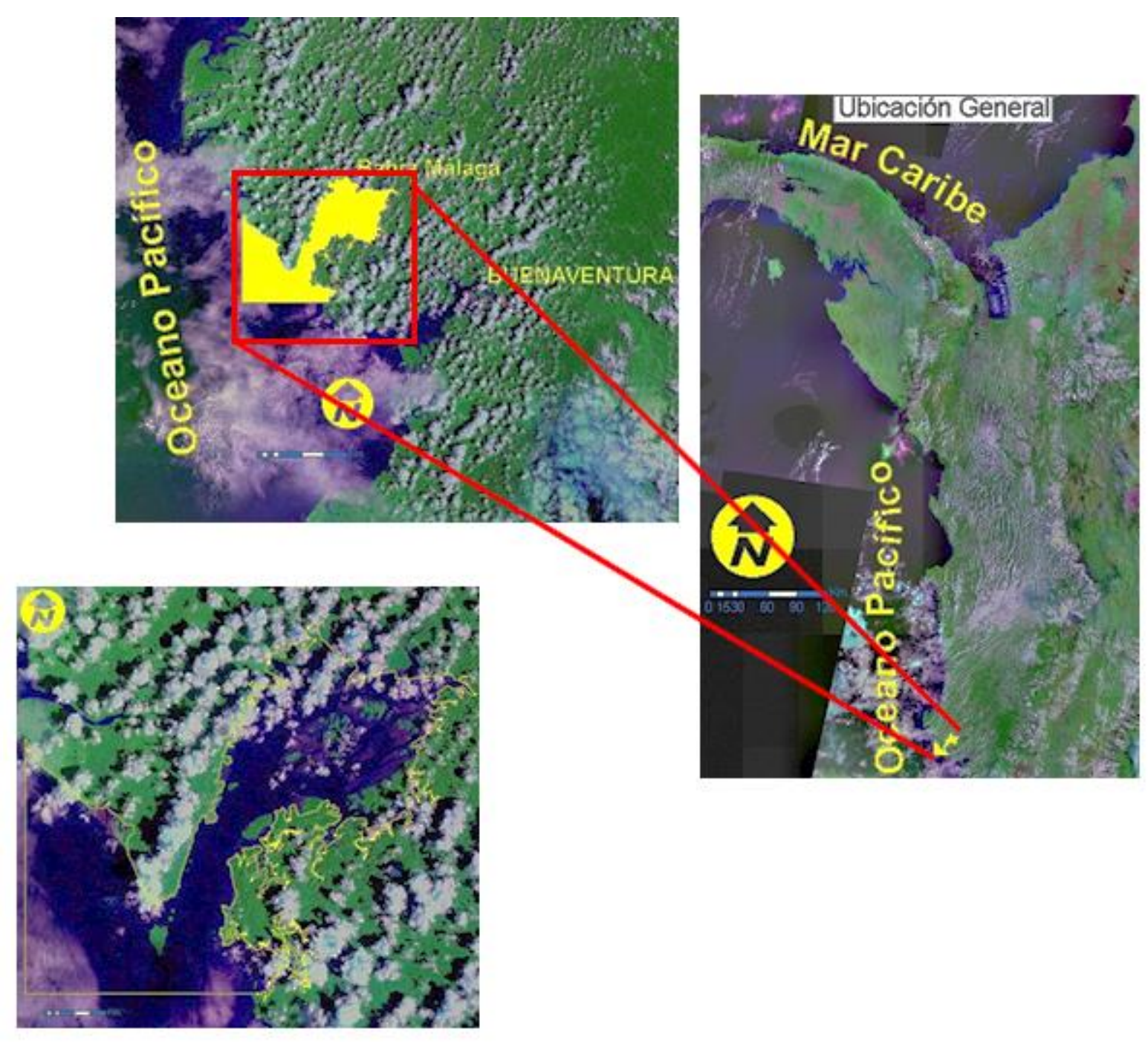

Figura 1: Localización del área de Bahía Málaga. (Fuente: Parques Nacionales 2008).

La economía de esta comunidad se desarrolla basada en diferentes actividades de subsistencia y productivas como son: la pesca, caza, corte de madera, recolección de piangua, agricultura de subsistencia y ecoturismo, estas actividades de acuerdo con la información aportada por la junta del Consejo Comunitario de la Plata -Bahía Málaga pueden arrojar niveles de ingreso promedio de $\$ 147675$ pesos.

De acuerdo con la información dada por los líderes del consejo comunitario de la Plata - Bahía Málaga la distribución del ingreso promedio es la siguiente: compra de los productos requeridos para complementar la alimentación (arroz, hortalizas, aceite, azúcar, sal) para épocas como diciembre en la compra de ropa.

Políticamente el consejo comunitario de la Plata Bahía Málaga, además de ser territorio colectivo, es un lugar donde se encuentran unidas tres estrategias de conservación: el Parque Regional Natural la Sierpe, el Distrito de Manejo Integrado la Plata y el Parque Natural Nacional Uramba; el territorio cuenta con diferentes Planes de Manejo que apuntan a la conservación de los recursos naturales y a la organización de sus comunidades en relación al aprovechamiento y uso de sus recursos. 


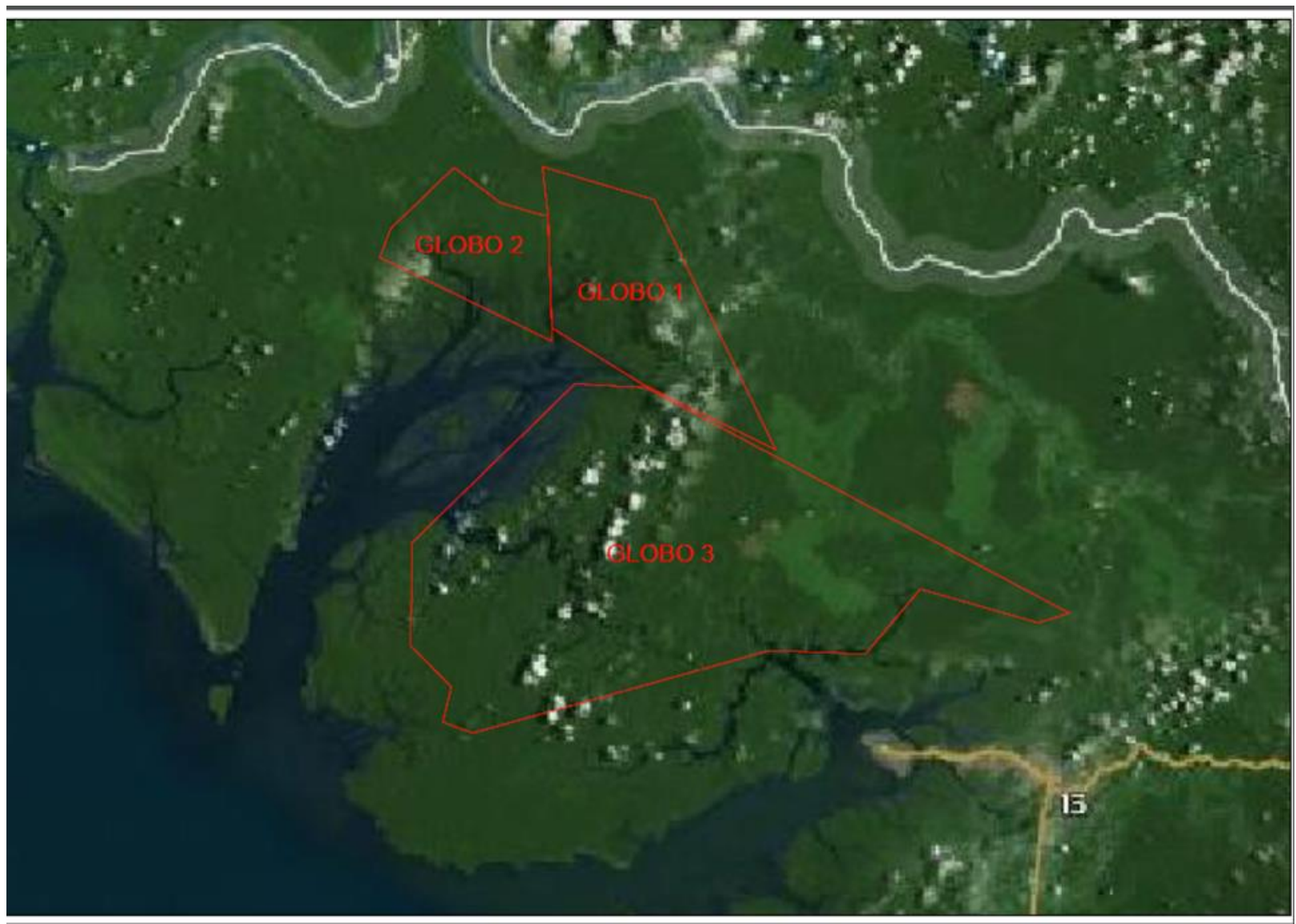

Figura 2: Plano de ubicación del polígono del territorio de la comunidad negra de la Plata Bahía Málaga.

La principal amenaza de este territorio, es la relacionada con los movimientos telúricos (sismos y tsunamis), esto debido a que esta zona se encuentra localizada sobre la fuente sísmica primaria y más importante del territorio colombiano, la zona de subducción del Pacífico. Por otra parte debido a las intensas lluvias y el poco manejo de las aguas de lavado y de escorrentía en la zona, se presentan amenazas por deslizamientos en algunas comunidades como el caso de Miramar y la Sierpe; en lugares como la Plata y Mangaña su afectación más recurrente se da por las continuas inundaciones, debido a que se encuentran a pocos metros sobre el nivel de las aguas. A estas amenazas naturales se les puede sumar, la pérdida de sus recursos pesqueros y de bosques por la explotación de propios y extraños y la constante preocupación, de que se renueve la propuesta de tiempo atrás de usar el territorio ancestral como una alternativa portuaria.

\section{CAMBIO CLIMÁTICO Y VULNERABILIDAD}

\section{Acercamiento conceptual al cambio climático}

Para la mayoría de las personas, la expresión "cambio climático" significa la alteración del clima mundial que nosotros, los seres humanos, estamos ocasionando como consecuencia del consumo de combustibles fósiles, la tala de bosques y otras prácticas que incrementan la concentración de gases de efecto invernadero (GEI) en la atmósfera.

Esta percepción concuerda con la definición oficial de la Convención Marco de las Naciones Unidas sobre el Cambio Climático (CMNUCC, o UNFCCC por sus siglas en inglés) en la que el cambio climático se puede atribuir "directa o indirectamente a las actividades humanas que alteran la composición de la atmósfera global y que se suma a la variabilidad del clima natural observada durante períodos de tiempo comparables". 
Sin embargo, con frecuencia los científicos utilizan el término cambio climático para señalar cualquier cambio en el clima, ya sea de origen natural o humano. En particular, el Grupo Intergubernamental de Expertos sobre el Cambio Climático (IPCC, por sus siglas en inglés) lo define como "un cambio en el estado del clima que se puede identificar... por cambios en el promedio y/o la variabilidad de sus propiedades y que persiste durante un período prolongado, generalmente decenios o períodos más largos".

\section{El tiempo y el clima}

El tiempo es una combinación de condiciones meteorológicas -viento, lluvia, nieve, sol, temperatura, etc., en un momento y lugar determinado, lo que configura la denominada "variabilidad climática".

En cambio, el término "clima" describe las características generales y a largo plazo del tiempo que se experimenta en un mismo lugar. Los ecosistemas, la agricultura, los medios de sustento y los asentamientos de una región dependen en gran medida de su clima. De allí que se pueda pensar que el clima es como un "resumen" a largo plazo de las condiciones del tiempo, tomando en cuenta tanto las condiciones promedio como la variabilidad de éstas (ONU \& EIRG 2008).

Las fluctuaciones que ocurren año tras año y las estadísticas de las condiciones extremas, tales como tormentas intensas o temporadas excepcionalmente calientes, forman parte de la variabilidad climática. Además, algunos fenómenos que cambian paulatinamente pueden perdurar durante estaciones enteras o hasta años.

El fenómeno más conocido en este sentido es El Niño. Debido a que la atmósfera conecta a todos los sistemas meteorológicos, a veces es útil describir a la atmósfera, los océanos y la superficie de la Tierra (ONU \& EIRG 2008).

El cambio climático es uno de los mayores desafíos que la humanidad deberá afrontar en el presente siglo. Amenaza el ambiente, empeorando las condiciones de vida de la población y retrasando el logro de sus metas de desarrollo en especial en aquellas comunidades más pobres, donde los impactos del cambio climático, aumentarían de manera exponencial los ya deteriorados sistemas económicos de estos sectores. (MAVDT et al. 2010).
Frente a esta realidad la Convención Marco de las Naciones Unidas sobre Cambio Climático - CMNUCC en el Artículo $4^{\circ}$ de la Convención recomienda que el compromiso más importante, es formular, aplicar, publicar y actualizar regularmente programas nacionales $\mathrm{y}$, según proceda, regionales, que contengan medidas orientadas a mitigar el cambio climático, teniendo en cuenta las emisiones antropógenas por las fuentes y la absorción por los sumideros de todos los gases de efecto invernadero no controlados por el Protocolo de Montreal, y medidas para facilitar la adaptación adecuada al cambio climático.

Es así como a partir de 2010 Colombia, en el marco del Plan de Nacional de Desarrollo 2010-2014 "Prosperidad para todos" empezó a trabajar cuatro estrategias para afrontar el cambio climático: el Plan Nacional de Adaptación al Cambio Climático - PNACC, la Estrategia Colombiana de Desarrollo Bajo en Carbono - ECDBC, la Estrategia Nacional para la Reducción de las Emisiones debidas a la Deforestación y la Degradación Forestal en los Países en Desarrollo; la Función de la Conservación, la Gestión Sostenible de los Bosques y el Aumento de las Reservas Forestales de Carbono en los Países en Desarrollo - ENREDD+ y la Estrategia de Protección Financiera ante Desastres. (CONPES 3700 2011).

Evidentemente el camino que han decidido tomar las autoridades nacionales sobre el cambio climático, está basado en las acciones de mitigación y adaptación, esta última como una de las estrategias que dinamizan las acciones de la sociedad de manera integral y redefine las políticas en todos sus componentes; sin embargo, para que estas estrategias cumplan su función de forma correcta, es necesario entender y conocer la vulnerabilidad de los sistemas en los cuales se sustenta la población que impacta el territorio.

\section{ACERCAMIENTO CONCEPTUAL A LA VULNERABILIDAD}

El IPCC (2007) define vulnerabilidad como: "el grado de susceptibilidad o de incapacidad de un sistema para afrontar los efectos adversos del cambio climático y, en particular, la variabilidad del clima y los fenómenos extremos. La vulnerabilidad dependerá del carácter, magnitud y rapidez del cambio climático al que esté expuesto un sistema, y de su sensibilidad y capacidad de adaptación". 
Esta última consiste en la habilidad de un sistema para ajustarse al Cambio Climático con la finalidad de moderar los daños, aprovechar oportunidades y enfrentar las consecuencias; además es de fundamental importancia ya que es el mecanismo que permite reducir la vulnerabilidad (TejedaMartínez \& Conde-Álvarez 2009).

Otra definición de la vulnerabilidad de una población o sistema, frente a los cambios climáticos se refiere al grado en que un sistema o asentamiento está expuesto a alguna amenaza climática y a la capacidad que tiene para manejar los daños (riesgos), sin que les afecte; es decir, los mecanismos de adaptación frente a los cambios climáticos.

Esta capacidad está relacionada con la manera como la población o el sistema son afectados, y con el tiempo de afectación. Para ello es necesario conocer la sensibilidad a los cambios; es decir, saber los efectos de las condiciones climáticas (sobre la población o el sistema) y cómo éstos responderán a los cambios (susceptibilidad) o sea su capacidad de adaptación.

De esta manera, la vulnerabilidad esta intrínsecamente relacionada con la amenaza a la que se está expuesto, y a la sensibilidad y capacidad de adaptación de quienes están expuestos (ONU/EIRG 2008)

Wilches (1998) menciona que la vulnerabilidad está claramente determinada por una serie de factores definidos así:

Factores Ambientales: Son aquellos que se relacionan con la manera de cómo una comunidad determina "explota" los elementos de su entorno, debilitándose a sí misma y debilitando a los ecosistemas en su capacidad para absorber sin traumatismos los fenómenos de la naturaleza (Wilches 1998).

Factores socio-económicos: Se refiere tanto a la ausencia de recursos económicos de los miembros de una comunidad (que los obliga, por ejemplo, a invadir zonas de amenaza o a construir sin la técnica o los materiales adecuados), como a la mala utilización de los recursos disponibles para una correcta "gestión del riesgo". La pobreza es quizás la principal causa de vulnerabilidad (Wilches 1998).

Factores Físicos: Son aquellos que tiene que ver, entre otros aspectos, con la ubicación física de los asentamientos o con las calidades y condiciones técnicas -materiales de ocupación o aprovechamiento del ambiente y sus recursos (Wilches 1998).

Factores sociales: Se refiere a un conjunto de relaciones, comportamientos, creencias, formas de organización (institucional y comunitaria) y maneras de actuar de las personas y las comunidades que las coloca en condiciones de mayor o menor exposición (Wilches 1998).

\section{METODOLOGÍA}

La metodología de esta investigación tuvo un enfoque mixto, empírico-analítica, puesto que se busca describir y analizar la percepción de vulnerabilidad de las comunidades del territorio del Consejo Comunitario de la Plata Bahía Málaga. Para ello se establecieron dos procesos, el primero consistió en la recolección y revisión de información secundaria relacionada con la vulnerabilidad de las comunidades rurales ante al cambio climático, incluyendo la información relacionada con las condiciones geofísicas, socioeconómicas, político institucional y culturales, del área de estudio.

El segundo proceso consistió en el trabajo de campo en el territorio colectivo del consejo comunitario de la comunidad negra de La Plata Bahía Málaga donde se tomó información directa mediante la utilización de dos técnicas de recolección de información (grupos focales y entrevistas semiestructurada). Estas técnicas de investigación se basan en las declaraciones emitidas por una muestra representativa de una población concreta, que nos permite conocer sus opiniones, actitudes, creencias, valoraciones subjetivas, conocimientos etc.

Para el desarrollo de esta técnica se utilizaron unas guías previamente diseñadas por el grupo de investigación, Medio Ambiente y Desarrollo Sostenible de la Universidad de Manizales, las cuales fueron utilizadas en el proyecto macro en otras regiones. Los participantes fueron seleccionados con el apoyo de los miembros de la junta directiva del Consejo Comunitario de la Comunidad Negra de La Plata -Bahía Málaga, donde se establecieron algunos criterios para su selección, como por ejemplo; tener cierto liderazgo en la comunidad, estar en la comunidad hace más de un año; tener conocimiento de las problemáticas de la comunidad, entre otros; con estos criterios se conformó un grupo compuesto por diferentes líderes de cada uno de las comunidades que 
componen el Consejo Comunitario (La Plata, Mangaña, La Sierpe, Miramar), es así que se contó, tanto con profesores, estudiantes, líderes de las diferentes comunidades, y líderes de la Junta directiva del Consejo Comunitario de la Plata Bahía Málaga, que residen en cada una de las comunidades del territorio del consejo. Se tuvo muy presente involucrar, tanto los líderes políticos del consejo comunitario, como los líderes naturales haciendo diferencia en las edades a fin de medir la percepción generacional. En este mismo grupo se encontró personas que desempeñan diferentes actividades económicas, como pesca, agricultura, recolección de piangua, corteros de madera; lo cual le dio más valor a los comentarios de los participantes.

Como estrategia complementaria, antes de la realización de la entrevista de grupo focal se realizó un ejercicio introductorio donde se hicieron aclaraciones de conceptos básicos, conceptos de participación, el grado de su importancia y la honestidad con que debe ser manejada la información a suministrar.

Los guiones de los instrumentos utilizados fueron adaptados a las características propias de la comunidad utilizando terminología sencilla y común, para hacer más fácil su entendimiento por todas las personas de la comunidad. Se realizaron entrevistas semiestructuradas a cuatro líderes de la comunidad, tres hombres y una mujer, para el caso del grupo focal se realizó una reunión donde asistieron once personas pertenecientes a las cuatro comunidades con edades entre los diecinueve y setenta años.

La reunión de grupo focal se realizó en la vereda de la Plata del Consejo Comunitario, en el mes de junio del año 2013.

\section{RESULTADOS Y DISCUSIÓN}

De acuerdo a lo mostrado en la caracterización del territorio del consejo comunitario de la Plata Bahía Málaga se evidencia que está expuesta a diferentes amenazas; condiciones como altura sobre el nivel del mar, localización, temperatura, pluviosidad, geología del terreno, dan una calificación alta de exposición a amenazas climáticas de carácter local o global, bien sea por inundaciones, deslizamientos, o eventos extremos como tsunami, vendavales o sismos, etc.

Las amenazas a las cuales se sienten más expuestos los habitantes de estas comunidades son: el aumento del nivel del mar, las fuertes lluvias, perdida de territorio por erosión costera y las presiones antrópicas sobre los recursos naturales de flora y fauna. En lo relacionado con el aumento del nivel del mar estas percepciones concuerdan con los planteamientos realizados por el IPCC en su tercera comunicación donde manifiesta que muchas zonas costeras experimentarán aumento de niveles de inundación, erosión acelerada, pérdida de humedales y de manglares, e intrusión de agua del mar en las fuentes de agua dulce como resultado del cambio climático. Así mismo los cambios del nivel relativo del mar variarán localmente, debido a ascensos o subsidencias causados por otros factores (IPCC 2001).

En esa misma línea el IDEAM manifiesta que el mar para el caso del Litoral Pacífico aumentara en $60 \mathrm{~cm}$ para el año 2060 (IDEAM 2001). Más específicamente en las lecturas de las estaciones mareográficas de Buenaventura (Valle del Cauca) y de otras estaciones comparables, muestran valores similares de ascenso del nivel del mar (aunque mayores en Buenaventura). La tendencia calculada sobre las series de datos en puntos costeros de Panamá y Ecuador, muestran incrementos entre 0,9 mm y 1,4 mm por año, mientras en Buenaventura el aumento es de 2,2 mm por año (IDEAM 2010).

Estos impactos afectaran no solo a los diversos ecosistemas costeros, sino también a los asentamientos de las costas y estados insulares en términos de vidas, salud, vivienda, infraestructura y producción; también se afectaran las actividades económicas relacionadas con el turismo y las dinámicas culturales de apropiación de espacios como las playas para procesos simbólicos o actividades productivas (Chavarro et al. 2008).

Como se puede apreciar, la percepción comunitaria es coherente con muchos de las predicciones que han realizado las instituciones encargadas de estos temas, y es un indicador importante el hecho que se reconozca de manera propia que se encuentran expuestos a estas amenazas.

De las cuatro comunidades existentes en el territorio del consejo comunitario, las localidades de Mangaña y la Plata, presentan una alta exposición a los efectos climáticos (aumento del nivel del mar y de eventos extremos como tsunamis y vendavales), debido a su ubicación en zonas bajas a orillas del mar; sin embargo las otras dos; Sierpe y Miramar, que se encuentran en zonas altas actualmente están muy expuestas a los deslizamientos, por el incremento en las lluvias y el 
deficiente manejo de las aguas de escorrentía del sector, sin sustraer su exposición a eventos como los vendavales o fuertes vientos.

Además del elemento geográfico territorial, la exposición de estas comunidades está relacionada con su entorno natural y uno de los ecosistemas importantes en este territorio es el referido al manglar. En estas comunidades se percibe el valor de este ecosistema no solo en la producción de bienes sino también en la sensación de seguridad que representa para los habitantes de esta zona; estos establecen una relación directa entre su presencia y la protección que les puede brindar frente a eventos extremos como tsunamis o grandes ventiscas

Pese a la importancia que tienen para las comunidades y otros ecosistemas, los manglares están siendo fuertemente intervenidos. En Colombia, según el informe de estado de los ambientes marinos y costeros, para el año 2004, los manglares tenían una extensión de 380634 ha. De los cuales 292726 ha, se encontraron en la costa Pacífica y 87908 en la costa Caribe (INVEMAR 2004), las principales amenazas a las que se ven enfrentados los manglares son el cambio climático (especialmente el aumento del nivel del mar), los desarrollos urbanísticos, la sobreexplotación de los recursos y los cambios en el uso del suelo (Harpern et al. 2007, Alongi 2008, Gilman et al. 2008,).

Precisamente las comunidades del C.C de la Plata Bahía Málaga expresan que la sobreexplotación de los árboles de manglar es una de las principales amenazas a las cuales se encuentra expuesto este ecosistema y las especies asociadas (piangua, cangrejo etc.). Así como lo expresa (Duke et al. 2007), las comunidades humanas que habitan cerca de los manglares pueden perder el acceso a fuentes esenciales de alimentos, fibras, madera, químicos y medicinas, al igual que se altera el soporte de las redes alimenticias terrestres y marinas, se pone en peligro la fauna cuyos hábitats están ligados a estos ecosistemas.

Debido a la condición rural de este territorio, donde los desarrollos económicos son nulos o inexistentes, las comunidades sustentan su supervivencia esencialmente en los recursos naturales, lo cual los hace supremamente sensibles a los cambios que el clima pueda tener

Las comunidades manifiestan que actualmente se presentan inundaciones con mayor frecuencia de los sitios que históricamente han sido utilizados como recolección de productos hidrobiológicos como la piangua, cangrejo y el piacüil entre otros. Estos sitios de gran importancia para la comunidad ya que parte de su economía se desarrolla basada en diferentes actividades productivas y de subsistencia, como son: la pesca, caza, corte de madera, recolección de piangua, agricultura de subsistencia y ecoturismo.

De acuerdo al IPCC la subida del nivel del mar afectará a los ecosistemas de manglares al eliminar sus actuales hábitat y crear nuevas zonas inundadas por las mareas a las que podrían desplazarse algunas especies de manglares (IPCC 2001). Este aumento destruye los lugares de pesca y de recolección, de especies importantes para la seguridad alimentaria de la comunidad. Por tanto al afectarse estos sectores, su supervivencia se ve seriamente golpeada, no solo en sus condiciones alimenticias sino en su concepción de territorio; y para estas comunidades esta concepción va más allá de un simple espacio donde asientan las estructuras.

La comunidad presenta una organización comunitaria reconocida, gracias a su conformación como consejo comunitario (ley 70 de 1993), sin embargo, no ha logrado una verdadera articulación que permita pensar y planificar en conjunto sus acciones frente a diferentes eventos, en particular los relacionados con las amenazas y riesgos, los entrevistados son conscientes de que normalmente la comunidad en términos de los cambios del clima no han realizado ninguna planificación; así como lo manifiesta uno de los entrevistados “....en la comunidad la costumbre es no realizar ninguna planificación ni anticipación frente a los cambios del clima, estos solo se afrontan cuando lleguen, bien sea cambiando de sitio o de actividad económica".

Es evidente que hay cierto grado de conocimiento en la comunidad frente a temas como tsunamis, sin embargo no se tiene un plan estructurado y practicado para afrontar un evento extremo; como lo manifiesta uno de los entrevistados"... no hay ningún comité de prevención de riesgos y desastres en el territorio, por lo tanto ellos no se encuentran preparados para enfrentarse a los peligros, es una situación difícil para los habitantes...".

En relación a documentos de planificación sobre el territorio, se pude comentar específicamente sobre dos: el Plan de Ordenamiento Territorial Municipal, POT y el Plan de manejo de los recursos naturales. Al revisar el Plan de Ordenamiento Territorial de Buenaventura, no se evidencia argumentación o 
algún ítem relacionado con el cambio climático, esto puede ser causa de lo nuevo del tema en el país, que solo recientemente inicia el análisis de esta problemática, sin embargo, la no inclusión en los planes demuestra la baja capacidad de la instituciones para atender el asunto referente al cambio climático especialmente en el área rural del Pacífico.

En la revisión realizada a los Planes de Manejo no se observa la inclusión de la dimensión de cambio climático que permita prospectar acciones de mitigación o reducción de la vulnerabilidad con relación a los efectos del cambio climático en los ecosistemas y en la población residente. En resumen en ninguno de estos instrumentos de planificación se habla de forma directa sobre la vulnerabilidad de las comunidades o de acciones de mitigación o adaptación, sin embargo si se hacen referencia a problemáticas ambientales y riesgos que se tienen en las comunidades, no obstante estas referencias no se refuerzan y se pierden en el tiempo y poco de esto se convierte en procesos que garanticen una adecuada preparación de las comunidades en estos temas.

Por otra parte según información de los lideres, en la comunidad no se evidencia la estructuración de un comité o un grupo de atención temprana a desastres naturales, a nivel municipal tampoco existen registros de que un grupo con estas funciones se encuentre conformado para esta comunidad, pese a la existencia por norma del Consejo Distrital de Gestión del Riesgo anteriormente llamado Comité Local de Prevención y Atención de Desastre, esto evidencia el grado de vulnerabilidad de la población.

A pesar del avance en el conocimiento de los ecosistemas presentes, tampoco se han hecho estudios relacionados que apunten a determinar cuál es grado de vulnerabilidad ecosistémico y mucho menos relacionarlos con los efectos de cambio climático.

La vulnerabilidad de las poblaciones rurales no se debe únicamente a las presiones de la naturaleza, sino a los procesos sociales, económicos y políticos a nivel local y global sobre los cuales incide el cambio climático (Flores-Moreno et al. 2011). El cambio tecnológico, el crecimiento demográfico, las presiones del mercado, los conflictos sociales y la guerra interna son algunos de los tantos otros procesos de origen antropogénico que pueden alterar las repercusiones de los eventos climáticos extremos en el largo plazo sobre las condiciones de vida de las poblaciones dependientes de los recursos naturales.
Asimismo, la presencia mayor o menor del estado y de una red social globalizada que interviene en los ámbitos de las comunidades, puede favorecer la acción colectiva y reducir la vulnerabilidad de la población local o, por el contrario, agudizar el conflicto cultural y poner en tela de juicio su poder sobre el territorio y su paz civil. (Blaikie et al. 1996).

Así, las fuerzas de la naturaleza ocupan una importancia secundaria en materia de vulnerabilidad, pues son las estructuras sociales y políticas las que influyen en la capacidad de agencia de la población para atenuar las adversidades (Blaikie et al. 1996).

Las actividades humanas ejercen una marcada influencia en la disminución del número de especies, en el tamaño y la variabilidad genética de las poblaciones silvestres y en la pérdida irreversible de hábitats y ecosistemas (Peña \& Neira, 1998). Para el caso de la comunidad de la Plata, las comunidades perciben los efectos de las actividades humanas en la pesca y relacionan sus causas en el uso de elementos de pesca muy destructivos que disminuyen sus reservas a niveles críticos

\section{CONCLUSIONES}

Pese al gran avance, en términos organizativos, logrados con la conformación del consejo comunitario, esta fortaleza no ha sido utilizada para enfrentar aspectos como el cambio climático y el entendimiento de la vulnerabilidad frente a las múltiples amenazas a las cuales están expuestos, el conocimiento de la comunidad en términos de vulnerabilidad es muy bajo y esto es perjudicial para ellos y aumenta el riesgo de ser afectados por un evento desastroso el cual provoque la pérdida de los pocos bienes que poseen.

Si bien los lideres, no tienen toda la conceptualización sobre la vulnerabilidad frente a la amenaza del cambio climático, si es claro para ellos sus efectos y se percibe gran compromiso a realizar actividades y acciones que ayuden a reducir los niveles de vulnerabilidad de la población, sin embargo para que esto sea posible debe ser continuo en el tiempo; es allí donde es necesario el apoyo de los entes territoriales, departamentales a fin de darle soluciones más practicas a los efectos del cambio climático en estas zonas pequeñas. Ahora bien los entrevistados manifiestan que el nivel de vulnerabilidad en la comunidad es medio, al analizar las condiciones de este 
territorio, se puede percibir claramente que esa percepción no es coherente con la realidad del territorio que habitan, si se observan los factores, organizativos, culturales, económicos, educativos y ambientales que están presentes, no es difícil concluir que la vulnerabilidad del sector es mucho mayor.

El amplio territorio, la baja densidad poblacional, construcciones flexibles entre otros, son indicadores de la gran capacidad adaptativa de esta comunidad, ya que frente a un evento de desastre estas condiciones les permitirían trasladarse fácilmente de un lugar a otro, sin afectar en gran medida sus condiciones anteriores. Sin embargo por los pocos recursos financieros, la poca fortaleza política, educativa, organizativa, el poco apoyo institucional; las tensiones a los recursos de flora y fauna, las presiones económicas hechas por los sectores poderosos a nivel nacional e internacional, los impactos culturales que deben soportar las comunidades y lo incierto de las condiciones climáticas en estos territorios, se esperaría que esta capacidad de adaptación sea menor y por lo tanto se incremente la vulnerabilidad de la comunidad frente a los efectos del cambio climático.

Evidentemente las expresiones dadas por la comunidad no constituyen una prueba suficiente de que estos efectos sean provocados por el cambio climático, y en muchos casos se puede decir que corresponden a consecuencias determinadas por efectos de variabilidad climática, sin embargo esta información aquí presentada es valiosa como información inicial para incorporarse a nuevas investigaciones al respecto, tales como el desarrollo de metodologías para la reducción de la vulnerabilidad en las comunidades rurales del Pacífico.

\section{AGRADECIMIENTOS}

Inicialmente agradecer a Dios por permitir la realización de este documento, seguidamente resaltar el apoyo del Consejo Comunitario de la Plata Bahía Málaga y sus líderes por su constante y activa participación; a la C.V.C, por su comprensión, a mi familia que soporto con entereza mis multiplex ausencias en el transcurso de este reto.

\section{REFERENCIAS}

ALONGI, D. 2008. Mangrove forests: Resilience, protection from tsunami and responses to global climate change. Estuarine, Coastal and Shelf Science 76: 1-13.

BLAIKIE, P. T. CANNON, I. DAVIS \& B. WISNER (eds) 1996. Vulnerabilidad: el entorno social, político y económico de los desastres. LA RED, Latino América. 290 pp.

CENIPACÍFICO - Centro de Investigaciones Marinas y Tecnológicas del Pacifico. 1986. “Impacto Ecológico en Bahía Málaga a raíz de los desarrollos de la Base Naval del Pacífico y carretera de acceso". Memoria Ejecutiva. Santiago de Cali, 230 pp.

CHAVARRO, M. GARCIA, A. GARCIA, J. PABON, J. D. PRIETO, A \& ULLOA, A. 2008. Preparándonos para el futuro; amenaza, riesgo, vulnerabilidad y adaptación al cambio climático. Material de difusión y socialización sobre cambio climático No 3.55 pp.

CONPES 3700. 2011. Departamento Nacional de Planeación, Consejo Nacional de Política Económica y Social República de Colombia. 21 pp.

CONSEJO COMUNITARIO DE LA PLATA BAHÍA MÁLAGA. 2012. Código de Régimen Interno y Reglamento de Uso y Manejo de los Recursos Naturales Territorio Ancestral de las Comunidades Negras del Consejo Comunitario de La Plata. Bahía Málaga, Pacífico colombiano. $72 \mathrm{pp}$.

CVC - CORPORACIÓN AUTÓNOMA REGIONAL DEL VALLE DEL CAUCA \& CONSEJO COMUNITARIO DE LA PLATA BAHÍA MÁLAGA. 2009. Plan de Administración y Manejo del Parque Natural Regional La Sierpe, Distrito de Manejo Integrado de la Plata Bahía Málaga. Buenaventura. Primera Edición. 41pp.

DANE - DEPARTAMENTO ADMINISTRATIVO NACIONAL DE ESTADISTICA. 2003. Censo poblacional. (Fecha de acceso Mayo de 2013). URL disponible en: http://www.DANE.gov.co/files/icer/2003/valle/t2.pdf.

DANE - DEPARTAMENTO ADMINISTRATIVO NACIONAL DE ESTADISTICA. 2005. Boletín Censo General 2005. Perfil Buenaventura. Valle del Cauca. (Fecha de acceso Mayo de 2013). URL disponible en: 
http://www.DANE.gov.co/files/censo2005/PERFIL_PDF_C G2005/76109T7T000.PDF. Consultado en Mayo 2013.

DECRETO 1745/1995 del 12 de octubre, Por el cual se reglamenta el Capítulo III de la Ley 70 de 1993, se adopta el procedimiento para el reconocimiento del derecho a la propiedad colectiva de las Tierras de las Comunidades Negras" y se dictan otras disposiciones. (Diario Oficial No 42.049, del 13 de octubre de 1995).

DUKE, N. MEYBECKE. J. DITTMANN, S. ELLISON, M. ANGER, K. BERGER, U. CANNICCI, S. DIELE, E. FIELD, C. KOEDAN, N. LEE, S. MARCHAND, C. NORDHAUS, I. \& DAHDOUH-GUEBAS, F. 2007. A world without mangroves? Science 6: 41-42.

FLORES, E. \& VALDIVIA, G. 2011. Las percepciones de la población rural campesina de la microcuenca Mollebamba sobre la incidencia del cambio climático en su forma de vida. Centro Bartolomé de Las Casas, serie; investigación Micro cuenca Mollebamba 5:8

GALINDO, G. PALACIOS, S. BERNAL, N. R. OTERO, J. \& BETANCOURTH, J. C. 2009. Planificación ecorregional para la conservación de la biodiversidad en el Pacífico continental colombiano. Serie Planificación Ecorregional para la Conservación de la Biodiversidad, Instituto de Investigación de Recursos Biológicos Alexander von Humboldt, Agencia Nacional de Hidrocarburos, The Nature Conservancy e Instituto de Hidrología, Meteorología y Estudios Ambientales 3: 24 .

GILMAN, E. ELLISON, J. DUKE, N. \& FIELD, C. 2008. Threats to mangroves from climatic change and adaptation options. Aquatic Botany 89:237-250.

HARPERN, B. SELKOE, K. MICHELI, F. \& KAPPEL, C. 2007. Evaluating and ranking the vulnerability of global marine ecosystems to anthropogenic threats. Conservation Biology 21:1301-1315.

IDEAM - INSTITUTO DE HIDROLOGÍA, METEOROLOGÍA Y ESTUDIOS AMBIENTALES. 2001. Vulnerabilidad y adaptación de la zona costera colombiana al ascenso acelerado del nivel del mar. Documento técnico de soporte para la primera comunicación de Colombia ante la convención marco de las naciones unidas sobre el cambio climático. 18 pp.
IDEAM - INSTITUTO DE HIDROLOGÍA, METEOROLOGÍA Y ESTUDIOS AMBIENTALES. 2010. Segunda Comunicación Nacional ante la Convención Marco de las Naciones Unidas sobre Cambio Climático. 34 pp.

IDEAM - INSTITUTO DE HIDROLOGÍA, METEOROLOGÍA Y ESTUDIOS AMBIENTALES. 2010. Nota Técnica. Cambio climático en temperatura, precipitación y humedad relativa para Colombia usando modelos meteorológicos de alta resolución (Panorama 2011-2100). 5 pp.

INVEMAR - INSTITUTO DE INVESTIGACIONES MARINAS Y COSTERAS. 2004. Informe de los Estado de los Ambientes Marinos y Costeros en Colombia en el año 2004 serie de publicaciones periódicas No. 8: 125

INVEMAR, UNIVALLE, E INCIVA. 2006. BIOMÁLAGA: Bases científicas y valoración de la biodiversidad marina y costera de Bahía Málaga (Valle del Cauca), como uno de los instrumentos necesarios para que sea considerada un área protegida. Cali, Colombia. 165 pp.

IPCC - INTERGOVERNMENTAL PANEL ON CLIMATE CHANGE. 2001. Cambio climático 2001: Tercer Informe de evaluación del Grupo Intergubernamental de Expertos sobre el Cambio Climático [IPCC, Ginebra, Suiza]. 12 pp.

IPCC - INTERGOVERNMENTAL PANEL ON CLIMATE CHANGE. 2007. Cambio climático 2007: Informe de síntesis. Contribución de los Grupos de trabajo I, II y III al Cuarto Informe de evaluación del Grupo Intergubernamental de Expertos sobre el Cambio Climático [Equipo de redacción principal: PACHAURI, R.K. \& REISINGER, A. (Directores de la publicación)]. IPCC, Ginebra, Suiza, 104 pp. URL disponible en: $\quad$ http://www.ipcc.ch/pdf/assessmentreport/ar4/syr/ar4_syr_sp.pdf

MAVDT - MINISTERIO DE AMBIENTE Y VIVIENDA Y DESARROLLO TERRITORIAL, IDEAM \& PROGRAMA DE LAS NACIONES UNIDAS PARA EL DESARROLLO -PNUD. 2010. Segunda comunicación Nacional ante la convención Marco de las Naciones Unidas sobre cambio Climático.

ONU/EIRG. 2008, El cambio Climático y la reducción del riesgo de desastres, nota informativa No. 1. 14 pp.

PEÑA, A. NEYRA, L. 1998. Amenazas a la biodiversidad. En: Conabio. La diversidad biológica de México: Estudio de País, 
1998: 158-180. Comisión Nacional para el Conocimiento y Uso de la Biodiversidad. Fernández Leal 43, Barrio de la Concepción Coyoacán, 04020 México, D.F.

TEJEDA-MARTÍNEZ A. \& CONDE-ÁLVAREZ, C. (eds.). 2009. Guía para la elaboración de Programas Estatales de Acción ante el Cambio Climático, PEACC, Instituto Nacional de Ecología, Universidad Veracruzana y Centro de Ciencias de la atmósfera UNAM. URL disponible en: http://www2.ine.gob.mx/sistemas/peacc/descargas/guias_ prog_est.pdf

WILCHES, G. 1998. Auge, caída y levantada de Felipe Pinillo, mecánico y soldador o yo voy a correr el riesgo. Guía de La Red para la gestión local del riesgo. URL disponible en: http://www.desenredando.org/public/libros/1998/gglr/in dex.html 